\title{
Thalidomide Prevents the Progression of Peritoneal Fibrosis in Mice
}

\author{
Hideyuki Arai ${ }^{1}$, Akira Furusu', Tomoya Nishino, ${ }^{1,2}$, Yoko Obata ${ }^{1,3}$, Yuka Nakazawa ${ }^{1}$, \\ Masayuki Nakazawa ${ }^{1}$, Misaki Hirose ${ }^{1}$, Katsushige Abe $^{4}$, Takehiko Koji ${ }^{2}$ and \\ Shigeru Kohno ${ }^{1}$
}

${ }^{1}$ Second Department of Internal Medicine, Nagasaki University School of Medicine, ${ }^{2}$ Department of Histology and Cell Biology, Nagasaki University Graduate School of Biomedical Sciences, Nagasaki, ${ }^{3}$ Career Development Center for Medical Doctors, Nagasaki University Hospital and ${ }^{4}$ Jinikai Hospital, Oita

Received October 18, 2010; accepted February 2, 2011; published online April 21, 2011

\begin{abstract}
Thalidomide is clinically recognized as a therapeutic agent for multiple myeloma and has been known to exert anti-angiogenic actions. Recent studies have suggested the involvement of angiogenesis in the progression of peritoneal fibrosis. The present study investigated the effects of thalidomide on the development of peritoneal fibrosis induced by injection of chlorhexidine gluconate (CG) into the mouse peritoneal cavity every other day for 3 weeks. Thalidomide was given orally every day. Peritoneal tissues were dissected out 21 days after CG injection. Expression of CD31 (as a marker of endothelial cells), proliferating cell nuclear antigen (PCNA), vascular endothelial growth factor (VEGF), $\alpha$-smooth muscle actin (as a marker of myofibroblasts), type III collagen and transforming growth factor (TGF) $\beta$ was examined using immunohistochemistry. CG group showed thickening of the submesothelial zone and increased numbers of vessels and myofibroblasts. Large numbers of VEGF-, PCNA-, and TGF- $\beta$-positive cells were observed in the submesothelial area. Thalidomide treatment significantly ameliorated submesothelial thickening and angiogenesis, and decreased numbers of PCNA- and VEGF-expressing cells, myofibroblasts, and TGF$\beta$-positive cells. Moreover, thalidomide attenuated peritoneal permeability for creatinine, compared to the CG group. Our results indicate the potential utility of thalidomide for preventing peritoneal fibrosis.
\end{abstract}

Key words: thalidomide, peritoneal fibrosis, PCNA, VEGF, TGF- $\beta$

\section{Introduction}

Peritoneal dialysis (PD) is a beneficial therapy for endstage renal disease in terms of preserving residual renal function and improving hemodynamic stability, but longterm PD therapy causes histopathological and functional changes in the peritoneum, such as peritoneal fibrosis with increased submesothelial collagen deposition, loss of mesothelial cells and ultrafiltration failure $[6,10,13]$. Williams et al. demonstrated a correlation between peritoneal vascular density and peritoneal fibrosis in patients on

Correspondence to: Akira Furusu, M.D., Ph.D., Second Department of Internal Medicine, Nagasaki University School of Medicine, 1-7-1 Sakamoto, Nagasaki 8528501, Japan.

E-mail: furusua-ngs@umin.ac.jp long-term PD [33]. Furthermore, Honda et al. [15] reported that the appearance of vasculopathy correlated with reductions in ultrafiltration. Other studies have shown that the concentration of vascular endothelial growth factor (VEGF) in peritoneal fluid is up-regulated in PD patients [37, 38]. These findings suggest that angiogenesis is also associated with the pathogenesis of peritoneal fibrosis and peritoneal dysfunction.

To elucidate the mechanism of peritoneal fibrosis, it would be useful to utilize convenient and reproducible experimental animal models [23, 35]. Of these, we used a mice model in the present study, which may be a more useful experimental model for studies examining immune system involvement in disease progression because of the availability of histopathological and cytological antibodies, therapeutic agents, and genetically engineered mice [17]. 
Although the mechanisms underlying the development of peritoneal fibrosis by chlorhexidine gluconate (CG) are unclear, research has shown that persistent chemical irritation by $\mathrm{CG}$ induces tissue damage with inflammation, followed by tissue repair with excessive proliferation of fibroblasts [23]. In addition, we have demonstrated that the number of blood vessels also increases during the progression of CG-induced peritoneal fibrosis [23, 27, 35]. These changes resemble those seen in the peritoneum of long-term PD patients.

Yoshio et al. recently demonstrated that TNP-470, an anti-angiogenic compound, markedly reduced the number of blood vessels and myofibroblasts and suppressed the progression of CG-induced peritoneal fibrosis in an experimental mouse model [35]. In addition, Io et al. demonstrated a reduction in peritoneal-associated vessels and suppression of peritoneal fibrosis by anti-VEGF neutralizing antibody injection in a rat peritoneal fibrosis model induced by $\mathrm{CG}$ injection [16]. These results suggest that anti-angiogenic therapies or drugs might limit or prevent the progression of peritoneal fibrosis in patients with long-term PD.

Thalidomide, $\alpha-\mathrm{N}$-phthalidoglutarimide $\left(\left(\mathrm{C}_{13} \mathrm{H}_{10} \mathrm{~N}_{2}\right)_{4}\right)$, was originally marketed as a sedative and anti-emetic, but was withdrawn following the identification of severe teratogenic effects [5]. The mechanisms of action for thalidomide are complex and probably include different molecular targets. D'Amato et al. have reported that thalidomide inhibits angiogenesis by interrupting processes mediated by basic fibroblast growth factor (bFGF) and/or VEGF [8]. Thalidomide may thus exert potent immunomodulatory, anti-angiogenic, and anti-proliferative actions. Indeed, the ability of thalidomide to inhibit angiogenesis was confirmed in a rabbit cornea micropocket assay [8]. Furthermore, the antifibrotic effects of thalidomide have been reported in animal models of bleomycin-induced pulmonary fibrosis [32], liver cirrhosis [19-21, 29], myocardial infarction [34], and peritoneal fibrosis [25].

The present study investigated the effects of thalidomide, which has anti-angiogenic and anti-proliferative actions, on the progression of peritoneal fibrosis using a mouse model of peritoneal fibrosis induced by CG injection.

\section{Materials and Methods}

\section{Animals}

Male ICR mice (Japan SLC, Shizuoka, Japan) at 8 weeks old (body weight, $25 \pm 3 \mathrm{~g}$ ) were used in the present study. Animals were housed in a light- and temperaturecontrolled room in the Biomedical Research Center of the Center for Frontier Life Sciences at Nagasaki University. They had free access to laboratory chow and tap water in standard rodent cages. Based on the national regulations and guidelines, all experimental procedures were reviewed by Institutional Animal Care and Use Committee of Nagasaki University and finally approved by the president (No. 0605220510).

\section{Experimental protocol}

Peritoneal fibrosis was induced by intraperitoneal injection of $0.1 \% \mathrm{CG}$ in $15 \%$ ethanol dissolved in saline, as described previously with some modifications [27, 35]. Mice received injections of CG into the peritoneal cavity at a volume of $10 \mathrm{~mL} / \mathrm{kg}$ body weight every other day for 3 weeks. Mice were divided into four groups $(n=5$ each). The CG group received injections of CG into the peritoneal cavity and oral vehicle (methylcellulose) 5 times/week for 3 weeks. The $\mathrm{CG}+$ thalidomide group received oral thalidomide (kindly donated by Fujimoto Pharmaceutical Corp., Osaka, Japan) 5 times/week with CG for 3 weeks. The control group was injected intraperitoneally with $15 \%$ ethanol in saline and given oral vehicle. The thalidomide group was injected intraperitoneally with $15 \%$ ethanol in saline and given oral thalidomide. Dose and interval for oral administration of thalidomide were selected based on pilot studies conducted to determine the effects of different doses on thickness of the submesothelial area. Thalidomide doses $<10 \mathrm{mg} / \mathrm{kg}$ did not reduce peritoneal thickness compared with untreated CG group, so we selected a dose of $10 \mathrm{mg} / \mathrm{kg}$ administered 5 times/week for the present study. Injection of $0.1 \% \mathrm{CG}$ was performed under ether anesthesia to ensure injection of the correct dose at the correct site, and to avoid induction of pain during intraperitoneal injection. Mice were sacrificed at 2 or 3 weeks just after the peritoneal function test, and peritoneal tissues were carefully dissected out. To avoid direct damage to the peritoneum caused by repeated injections, $\mathrm{CG}$ injections were made into the lower part of the peritoneum, while the upper portion of the parietal peritoneum was used for examination. Tissues were fixed with $4 \%$ paraformaldehyde immediately after sampling and embedded in paraffin.

\section{Histological and immunohistochemical examination}

For morphological examination, $4-\mu \mathrm{m}$ thick paraffinembedded tissues were stained with hematoxylin and eosin (HE). The immunohistochemical procedure used in the present study was described previously $[1,31]$. The following antibodies were used for immunohistochemistry: 1) goat anti-mouse CD31 antibody diluted 1/50 (sc-1506; Santa Cruz Biotechnology, Santa Cruz, CA, USA), as a marker for endothelial cells; 2) mouse anti-proliferative cell nuclear antigen (PCNA) antibody diluted 1/200 (M0879; Dako, Glostrup, Denmark), as a marker for proliferative cells; 3 ) mouse anti-human $\alpha$-smooth muscle actin (SMA) antibody diluted 1/100 (M0851; Dako), as a marker for myofibroblasts; 4) rabbit anti-human transforming growth factor (TGF)- $\beta$ antibody diluted 1/100 (sc-146; Santa Cruz Biotechnology); 5) rabbit anti-rat type III collagen antibody diluted 1/400 (LB1393; LSL, Tokyo, Japan); and 6) rabbit anti-human VEGF antibody diluted 1/50 (sc-507; Santa Cruz Biotechnology).

To block endogenous peroxidase, renal sections were reacted with methanol containing $0.3 \% \mathrm{H}_{2} \mathrm{O}_{2}$ for 15 min at room temperature. An indirect immunohistochemical technique was used for PCNA, type III collagen and $\alpha$-SMA. 
For PCNA, deparaffinized tissue sections were incubated for 30 min with a blocking buffer containing normal goat immunoglobulin G (IgG) (I9140; Dako) diluted $1 / 20$ by $1 \%$ bovine serum albumin (BSA) in phosphate-buffered saline (PBS), then reacted with anti-PCNA antibody in $1 \% \mathrm{BSA} /$ PBS for $2 \mathrm{hr}$ at room temperature. After reacting with primary antibody, sections were reacted with horseradish peroxidase (HRP)-conjugated goat anti-mouse immunoglobulin antibody (P0447; Dako) diluted 1/100 for $1 \mathrm{hr}$ at room temperature. For type III collagen, deparaffinized tissue sections were incubated for 30 min with a blocking buffer containing 5\% normal goat serum, 5\% fetal calf serum, 5\% BSA, and 20\% normal swine serum in PBS. Sections were then reacted with the primary antibody diluted in the same blocking buffer. After reacting with anti-type III collagen antibody for $1 \mathrm{hr}$ at room temperature, sections were reacted with HRP conjugated swine antirabbit immunoglobulin antibody (P0399; Dako) diluted $1 / 50$ for $30 \mathrm{~min}$ and at room temperature, and peroxidase anti-peroxidase antibody (Z0113; Dako) diluted 1/100 for $30 \mathrm{~min}$ at room temperature. For $\alpha$-SMA, sections were reacted with a complex of anti- $\alpha$-SMA antibody and HRPconjugated rabbit anti-mouse immunoglobulin antibody (Z0109; Dako) diluted 1/100 for $1 \mathrm{hr}$ at room temperature, and HRP-labeled dextran polymer-conjugated goat antirabbit immunoglobulin antibody (K4002; Dako) for 30 min at room temperature. For CD31, VEGF and TGF- $\beta$, we applied the peroxidase and avidin-biotin-complex technique using Vectastain Elite ABC kit (Vector Laboratories, Burlingame, CA, USA), after reacting with primary antibodies for $16 \mathrm{hr}$ at $4{ }^{\circ} \mathrm{C}$. Reaction products were visualized by treating sections with $\mathrm{H}_{2} \mathrm{O}_{2}$ and 3,3'-diaminobenzidine tetrahydrochloride (DAB). Finally, sections were counterstained with methyl green and mounted. For all specimens, negative controls were prepared with irrelevant mouse monoclonal antibody, rat monoclonal antibody, normal goat $\mathrm{IgG}$, or normal rabbit IgG in place of the primary antibody.

\section{Terminal deoxynucleotidyl transferase (TdT)-mediated dUTP nick end-labeling (TUNEL) staining}

To identify apoptotic cells, TUNEL was performed according to the method of Gavrieli et al. [14] with a slight modification [9]. Paraffin sections (5-6 $\mu \mathrm{m}$ in thickness) on silane-coated glass slides were dewaxed and digested with $10 \mu \mathrm{g} / \mathrm{ml}$ of proteinase $\mathrm{K}$ in PBS at $37^{\circ} \mathrm{C}$ for $15 \mathrm{~min}$. The sections were then incubated with TdT buffer ( $25 \mathrm{mM}$ Tris/ $\mathrm{HCl}$ buffer, $\mathrm{pH} 6.6$, containing $0.2 \mathrm{M}$ potassium cacodylate and $0.25 \mathrm{mg} / \mathrm{ml} \mathrm{BSA}$ ) at room temperature for $30 \mathrm{~min}$. After incubation, the slides were reacted with $800 \mathrm{U} / \mathrm{ml}$ of TdT dissolved in TdT buffer supplemented with $0.5 \mu \mathrm{M}$ biotin-16-dUTP, $20 \mu \mathrm{M}$ dATP, $1.5 \mathrm{mM} \mathrm{CoCl}_{2}$ and $0.1 \mathrm{mM}$ dithiothreitol at $37^{\circ} \mathrm{C}$ for $90 \mathrm{~min}$. After washing with double-distilled water, signals were detected immunohistochemically using HRP-conjugated goat anti-biotin antibody. HRP sites were visualized with a mixture of DAB, hydrogen peroxide, nickel and cobalt ions.

\section{Peritoneal function test (PET)}

A PET was used to investigate peritoneal permeability parameters in mice, as described previously [26]. After anesthesia, a silicon catheter was inserted into the peritoneal cavity and $8.0 \mathrm{ml}$ of a standard dialysate (Dianeal ${ }^{\circledR}$; Baxter International Inc., Deerfield, IL, USA) containing 2.5\% glucose was instilled. Blood and dialysate samples were taken at $60 \mathrm{~min}$ after administration of dialysate. Transport of small solutes was evaluated using the dialysate-to-plasma $(\mathrm{D} / \mathrm{P})$ ratio of creatinine.

\section{Morphometric analysis}

We used image analysis software (Lumina Vision, Mitani Corp., Tokyo, Japan) on digitized images to assess the extent of peritoneal thickening. We measured the thickness of the submesothelial compact zone above the abdominal muscle in cross-sections of the abdominal wall. The image was transformed into a matrix of $1280 \times 1000$ pixels and viewed at $\times 200$ magnification. We selected a width of $840 \mu \mathrm{m}$ in the field examined under the microscope and measured the area of the submesothelial layer within the selected width of $840 \mu \mathrm{m}$. For each sample, 10 such areas were selected and the average area of the submesothelial layer was determined. In each peritoneal sample, numbers of CD31-positive vessels, PCNA-positive cells, VEGF-expressing cells, $\alpha$-SMA-expressing cells, and TGF$\beta$-expressing cells, and were counted in 10 fields at $\times 200$ magnification.

\section{Statistical analysis}

Data are expressed as mean \pm standard error of the mean (SEM). Differences among groups were examined for statistical significance using repeated-measures analysis of variance and the Bonferroni/Dunn test. Values of $P<0.05$ denoted the presence of a statistically significant difference.

\section{Results}

\section{Morphological examination}

Morphological changes were assessed by HE staining. In normal mice, peritoneal tissue comprises a peritoneal mesothelial monolayer and sparse connective tissues under the mesothelial layer (Fig. 1A). CG injection gradually induced exacerbation of peritoneal fibrosis (Fig. 1B, C). At 3 weeks, peritoneal samples from mice in the CG group showed marked thickening of the submesothelial compact zone and the presence of numerous cells (Fig. 1C). In the $\mathrm{CG}+$ thalidomide group, the thickness of the submesothelial compact zone and the number of infiltrating cells were significantly less than in the CG group (Fig. 1D). Peritoneal tissues in control mice were almost normal without any thickening of the submesothelial zone (data not shown). Repeated administration of thalidomide alone did not affect the thickening of the peritoneum compared with controls (data not shown). In addition, the severity of submesothelial area thickening in mice injected with $C G$ and the vehicle used to dissolve thalidomide was similar to that in CG- 

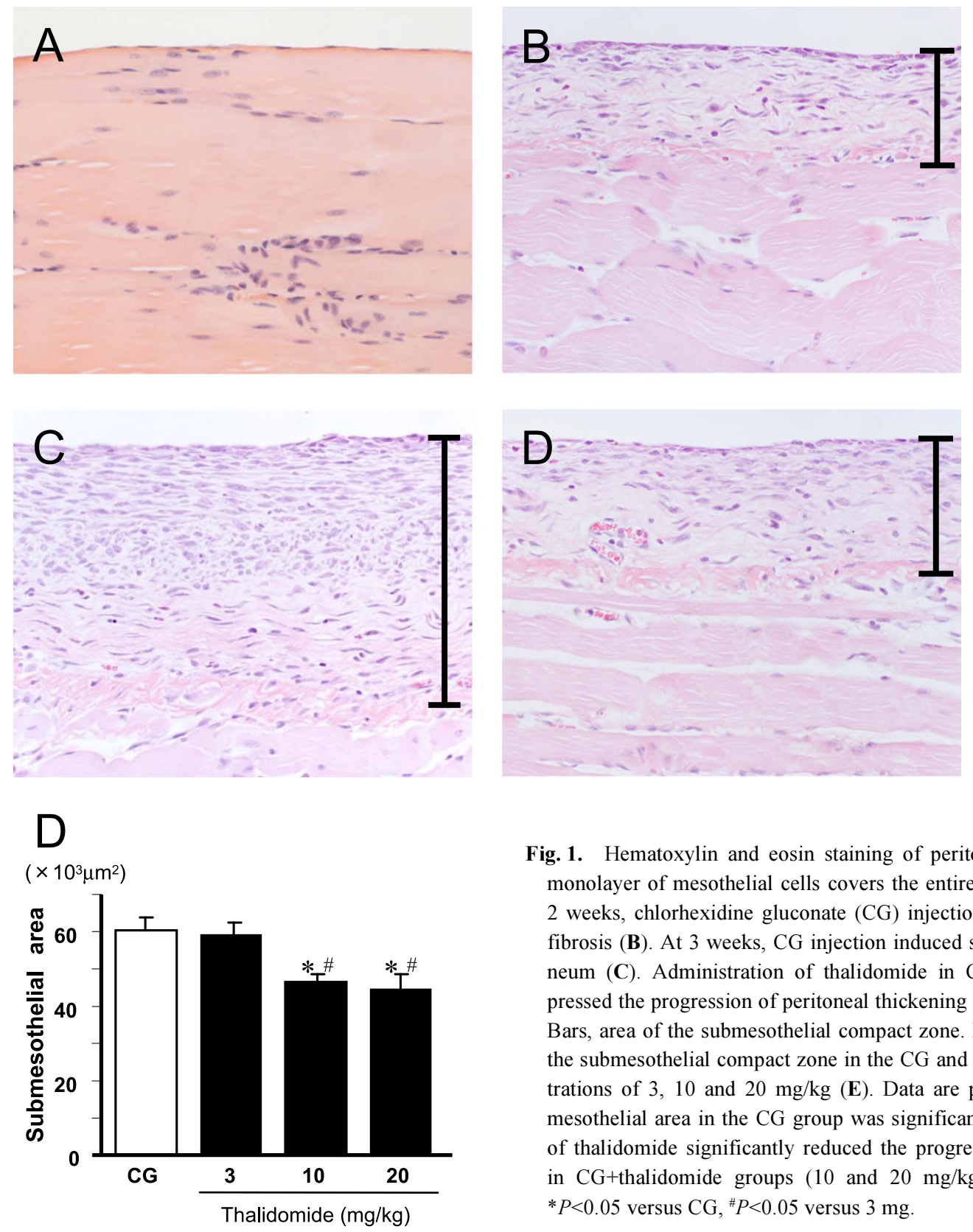

Fig. 1. Hematoxylin and eosin staining of peritoneal tissues. In normal mice, the monolayer of mesothelial cells covers the entire surface of the peritoneum (A). At 2 weeks, chlorhexidine gluconate (CG) injection has gradually induced peritoneal fibrosis (B). At 3 weeks, CG injection induced significant thickening of the peritoneum (C). Administration of thalidomide in CG-treated mice significantly suppressed the progression of peritoneal thickening at a concentration of $10 \mathrm{mg} / \mathrm{kg}(\mathbf{D})$. Bars, area of the submesothelial compact zone. Magnification: $\times 100$. Mean area of the submesothelial compact zone in the CG and CG+thalidomide groups at concentrations of 3,10 and $20 \mathrm{mg} / \mathrm{kg}(\mathbf{E})$. Data are presented as the mean \pm SEM. Submesothelial area in the $\mathrm{CG}$ group was significantly increased, while administration of thalidomide significantly reduced the progression of submesothelial thickening in $\mathrm{CG}+$ thalidomide groups (10 and $20 \mathrm{mg} / \mathrm{kg}$ ) compared with the $\mathrm{CG}$ group. * $P<0.05$ versus $C G$, ${ }^{\#} P<0.05$ versus $3 \mathrm{mg}$.

injected mice (data not shown). The results of semiquantitative analysis of the area of the submesothelial compact zone in each group are shown in Figure 1E.

\section{Expression of CD31}

Recent studies have described the presence of an increased number of vessels in thickened peritoneal tissues of both peritoneal dialysis patients [33] and experimental models of peritoneal fibrosis [35]. Meanwhile, several recent studies have indicated that thalidomide has antiangiogenic actions. To examine the effect of thalidomide on the vasculopathy observed in peritoneal fibrosis model, the number of blood vessels was quantified using immunohistochemistry for CD31. The number of CD31-positive vessels was markedly increased in mice from the CG group (Fig. 2A). In contrast, thalidomide administration decreased the number of CD31-expressing vessels (Fig. 2B). Table 1 summarizes the results of quantitative analysis of CD31positive vessels in the submesothelial compact zone.

\section{Expression of PCNA}

To examine whether thalidomide exerts anti-proliferative effects on the process of peritoneal fibrosis, PCNA staining was performed. Treatment with $\mathrm{CG}$ resulted in the appearance of numerous PCNA-positive cells in the thickened submesothelial area (Fig. 2C). Those cells comprised peritoneal mesothelial cells, fibroblast-like spindleshaped cells, and vascular endothelial cells. Compared with 

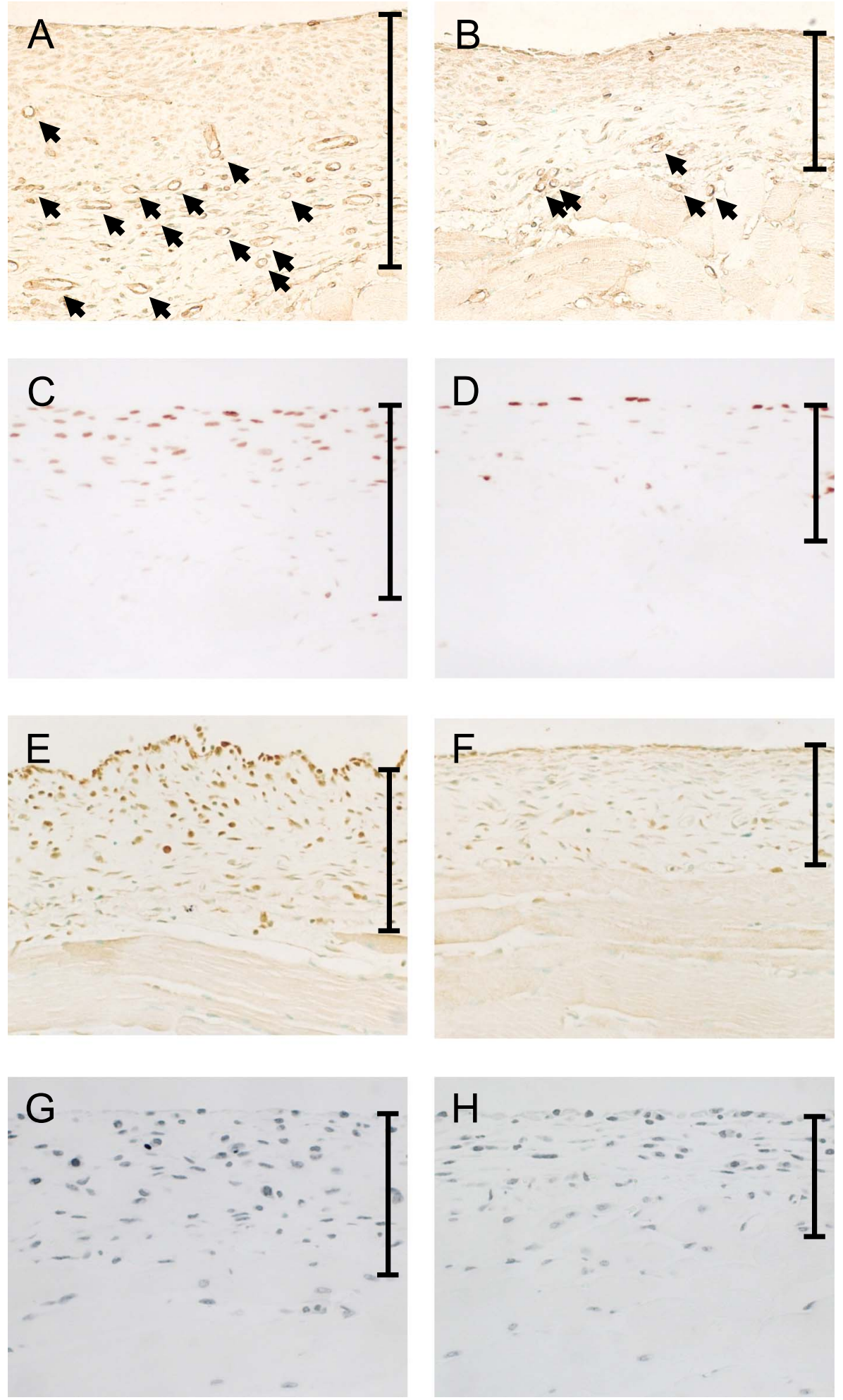

Fig. 2. Immunohistochemistry for CD31, PCNA, VEGF, and TUNEL. Note the presence of numerous vessels positively stained for CD31 (arrows) in the CG group (A), while few vessels are stained for CD31 (arrows) in the CG+thalidomide group (B). Magnification: $\times 200$. Note the presence of cells staining positively for PCNA in the CG group (C), while few cells stain for PCNA in the CG+thalidomide group (D). Magnification: $\times 200$. Note the large number of VEGF-expressing cells in the CG group $(\mathbf{E})$. Thalidomide treatment markedly decreased the number of these cells $(\mathbf{F})$ in the thickened submesothelial zone. Magnification: $\times 200$. Note the presence of TUNEL positive cells in the CG group and $\mathrm{CG}+$ thalidomide group $(\mathbf{G}$ and $\mathbf{H})$. The number of TUNEL positive cells in the CG+thalidomide group was as same as that in the CG group. Magnification: $\times 200$. 
Table 1. Number of positive cells of immunohistochemistry for CD31, PCNA, VEGF, $\alpha-S M A$, and TGF- $\beta$ in the peritoneum

\begin{tabular}{lcc}
\hline & CG group & CG+thalidomide group \\
\hline CD31 & $21 \pm 3$ & $4 \pm 1^{\mathrm{a}}$ \\
PCNA & $19 \pm 3$ & $11 \pm 2^{\mathrm{a}}$ \\
VEGF & $103 \pm 9$ & $65 \pm 6^{\mathrm{a}}$ \\
$\alpha-S M A$ & $61 \pm 4$ & $41 \pm 4^{\mathrm{a}}$ \\
TGF- $\beta$ & $148 \pm 7$ & $89 \pm 5^{\mathrm{a}}$ \\
\hline
\end{tabular}

Data are given as mean \pm SEM. The numbers of CD31-positive vessels, VEGF-expressing cells, PCNA-positive vessels, $\alpha$-SMA-expressing cells, and TGF- $\beta$-expressing cells were determined in 10 fields of the submesothelial region selected at random in each mouse and examined at $\times 200$ magnification. ${ }^{a} P<0.05$ versus $C G$ group

the CG group, treatment with thalidomide significantly reduced the number of PCNA-expressing cells, particularly vascular endothelial cells (Fig. 2D).

\section{Expression of $V E G F$}

Previous investigations have suggested the involvement of VEGF in the vasculopathy seen with peritoneal fibrosis [7, 16]. To examine the mechanisms of action for thalidomide on vasculopathy, the expression of VEGF in the CG group was compared with that in the $\mathrm{CG}+$ thalidomide group. In the control group, VEGF was expressed in the peritoneal mesothelial monolayer (data not shown). In the CG group, the number of VEGF-expressing cells was markedly increased compared with controls (Fig. 2E). Conversely, peritoneal tissues from the $\mathrm{CG}+$ thalidomide group contained fewer VEGF-expressing cells compared with the CG group (Fig. 2F). Table 1 summarizes the results of quantitative analysis of VEGF-positive vessels in the submesothelial compact zone.

\section{Involvement of apoptosis}

To clarify the involvement of apoptosis in CG-induced peritoneal fibrosis, TUNEL staining was performed. The number of TUNEL-positive cells was not different between the $\mathrm{CG}$ and $\mathrm{CG}+$ thalidomide groups. (Fig. $2 \mathrm{G}, \mathrm{H}$ ).

\section{Expression of $\alpha-S M A$ and type III collagen}

The results of immunohistochemical analyses for $\alpha$ SMA and type III collagen are shown in Figure 3 and Table 1. In the control group, expression of $\alpha$-SMA was only observed in vascular smooth muscle cells (data not shown). In contrast, the CG group showed $\alpha$-SMA expression in myofibroblasts in addition to vascular smooth muscle cells, and numerous $\alpha$-SMA-expressing myofibroblasts were identified in the thickened peritoneal tissues (Fig. 3A). Compared with the CG group, the number of $\alpha$-SMA-positive myofibroblasts was markedly reduced in the $\mathrm{CG}+$ thalidomide group (Fig. 3B). We also investigated peritoneal expression of type III collagen by immunohistochemistry. In the CG group, type III collagen was diffusely expressed in the submesothelial zone (Fig. 3C), while thalidomide treatment reduced type III collagen expression (Fig. 3D).

\section{Expression of TGF- $\beta$}

TGF- $\beta$ is widely known as an important mediator for progressing fibrosis. In addition, a previous report showed that increased expression of TGF- $\beta$ was associated with the extent of peritoneal fibrosis [22]. To examine whether thalidomide influenced the expression of TGF- $\beta$, the expression of TGF- $\beta$ was examined immunohistochemically. The number of TGF- $\beta$-positive cells was increased in the thickened peritoneum in the CG group (Fig. 3E), but was markedly reduced in the $\mathrm{CG}+$ thalidomide group (Fig. $3 \mathrm{~F}$, Table 1).

\section{Peritoneal transport study}

To investigate the effects of thalidomide on transport across the peritoneum, PET adapted for mice was evaluated [26]. CG treatment induced a major increase in permeability for creatinine, compared to that in control mice. In contrast, this modification was significantly attenuated with thalidomide treatment (Fig. 4).

\section{Discussion}

The present study shows that administration of thalidomide attenuates both CG-induced angiogenesis and CGinduced collagen accumulation in the thickened submesothelial area in an experimental mouse model of peritoneal fibrosis. Moreover, peritoneal transport studies demonstrated that small solute transport was promoted in the $\mathrm{CG}$ group, and that this increase in permeability was inhibited by thalidomide administration. These findings indicate that thalidomide attenuates both structural and functional damage of peritoneum in peritoneal fibrosis induced by CG and that thalidomide is potentially useful for preventing peritoneal fibrosis.

Concerning the mechanisms by which thalidomide inhibits angiogenesis in this model, analysis has focused on the effect of thalidomide on the expression of VEGF. VEGF is known to play an important role in endothelial cell proliferation, vascular permeability, and angiogenesis in several inflammatory lesions and tumors $[13,24]$. To date, VEGF expression has been confirmed to be up-regulated by various stimuli, such as low oxygen tension, cytokines, and TGF- $\beta$ $[11,12]$. Furthermore, several studies have shown that the concentration of VEGF in peritoneal fluid is increased in PD patients [38]. The present investigation demonstrated increased expression of VEGF in the submesothelial compact zone for the CG group compared with the control group, and this increase was significantly attenuated in the CG+thalidomide group. These results suggest that, in the present model, angiogenesis was attenuated through the inhibition of VEGF expression by thalidomide.

With respect to the inhibitory effect of thalidomide on CG-induced collagen accumulation, we must focus attention on the inhibitory effects of thalidomide both on the prolifer- 

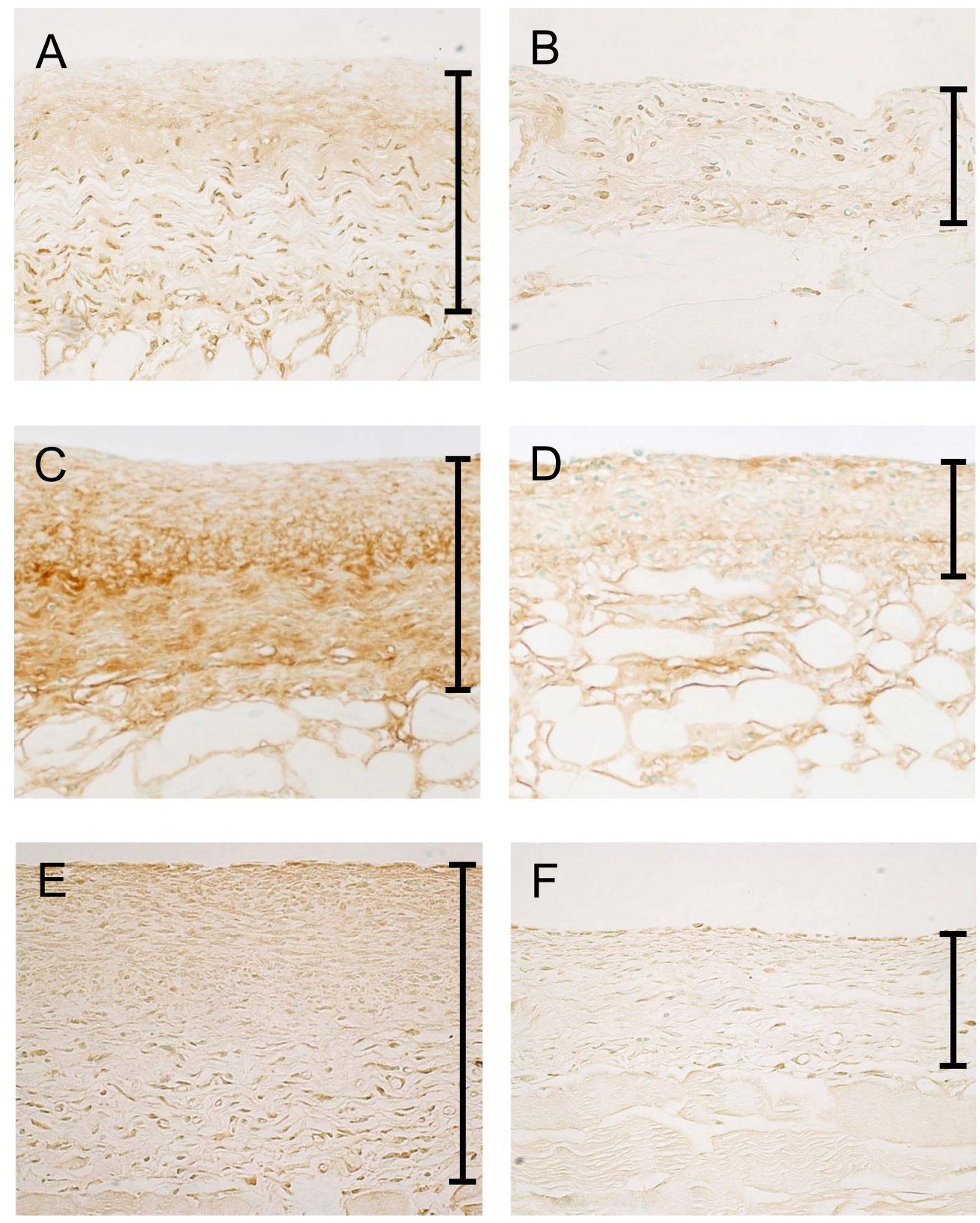

Fig. 3. Immunohistochemistry for $\alpha$-SMA, type III collagen, and TGF- $\beta$. In the CG group, note the presence of a large number of $\alpha$-SMApositive cells in the submesothelial zone (A). The number of $\alpha$-SMA-positive cells is reduced in the CG+thalidomide group (B). In the CG group, type III collagen is strongly expressed in the submesothelial compact zone (C), but is clearly decreased in the CG+thalidomide group (D). Note the strong expression of TGF- $\beta$ in the thickened submesothelial in the CG group (E), while such expression is decreased in the CG+thalidomide group $(\mathbf{F})$. Magnification: $\times 200$.

ation of myofibroblasts and on the expression of TGF- $\beta$. Several studies have demonstrated that TGF- $\beta$ is a profibrotic factor that increases fibroblast proliferation, stimulates the synthesis and deposition of extracellular matrix (ECM), and inhibits ECM breakdown [30]. In addition, TGF- $\beta$ promotes epithelial-to-mesenchymal transition (EMT) [18] and transdifferentiation of fibroblasts into myofibroblasts, which are the main cells producing collagen [30]. Our results showed that the number of TGF- $\beta$-positive cells decreased in the $\mathrm{CG}+$ thalidomide group together with a reduction in
" $\alpha$-SMA-positive" myofibroblasts and collagen accumulation in the submesothelial area. These data support the hypothesis that thalidomide inhibits collagen accumulation by anti-proliferative effects on myofibroblasts and by downregulatory effects on the expression of TGF- $\beta$ in myofibroblasts. In addition, the expression of heat shock protein 47 (HSP47), a molecular chaperone that is essential for collagen secretion and formation, was increased in the CG group but was reduced by thalidomide administration (data not shown). Moreover, thalidomide has been reported to 


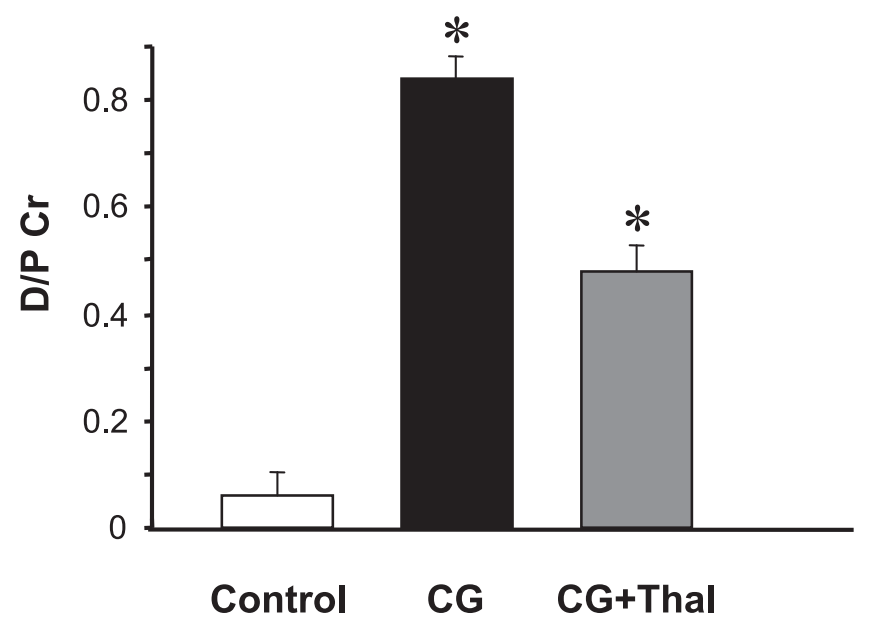

Fig. 4. Transport study. CG treatment induced a major increase in the permeability for creatinine. In contrast, this modification was attenuated with thalidomide treatment.

suppress expression of metalloproteinase (MMP)-2 and MMP-9, which are involved in collagen degradation, in vitro and in melanoma tumors [36]. Based on the above, the mechanism by which thalidomide attenuates CG-induced type III collagen accumulation was thought to involve not the promotion of collage degradation, but a decrease in collagen synthesis as well as suppression of collagen secretion and formation associated with reduced TGF- $\beta$ and HSP47 expression.

The various factors investigated herein using the enzyme-labeled antibody technique showed different distributions between the shallow and deep areas of the submesothelial compact zone. CG administration resulted in increased expression of vascular endothelial cells (CD31positive cells), myofibroblasts, and TGF- $\beta$-positive cells in the deep area of the submesothelial compact zone, while the number of these cells decreased following thalidomide administration. With regard to the mechanisms underlying these changes, we investigated the possibility of apoptosisinducing effects of thalidomide using the TUNEL method. However, as no differences were observed in the number of TUNEL-positive cells, the involvement of apoptosis was ruled out. Therefore, it was conjectured that the inhibitory effect of thalidomide on angiogenesis and myofibroblast proliferation observed in the present study was attributable to suppression of VEGF and TGF- $\beta$ expression caused by the inhibition of nuclear factor (NF)- $\kappa B$ activation by thalidomide [25]. Meanwhile, PCNA- and VEGF-positive cells were distributed in the shallow area of the submesothelial compact zone in the CG group. Previously, it was reported that TGF- $\beta$, TNF- $\alpha$, and other inflammatory cytokines produced by $\mathrm{CG}$ administration resulted in differentiation of mesothelial cells into highly proliferative mesenchymal cells and that these transdifferentiated cells increase the expression of VEGF [2]. Furthermore, it is well known that thalidomide exerts its anti-inflammatory effect through the inhibition of EMT inducers such as TGF- $\beta$ and TNF- $\alpha$ [28]. These findings suggested that the induction of EMT on mesothelium might contribute to distribution of PCNA- and VEGF-positive cells near the surface of the peritoneum in the CG group and decreases in the numbers of the aforementioned cells in the thalidomide group.

More recently, Mondello et al. demonstrated that treatment with thalidomide reduced the degree of peritoneal fibrosis in a rat model of peritoneal fibrosis induced by CG. They also showed that thalidomide reduced the expression of VEGF and TGF- $\beta$ in thickened peritoneal tissue and inhibited NF- $\kappa \mathrm{B}$ activation [25]. Concerning the anti-fibrotic effects of thalidomide, our results are in line with those reported by Mondello et al. However, the amount of thalidomide used in our study $(10 \mathrm{mg} / \mathrm{kg} /$ day $)$ was substantially smaller than that used in the study reported by Mondello et al. (100 mg/kg orally, 3 times/week). In addition, the effects of thalidomide treatment on peritoneal function have yet to be clarified in the previous paper [25].

From the perspective of safety, thalidomide can have severe adverse effects in a dose-dependent manner. In general, most animal experiments, including that by Mondello et al., have required relatively high doses (100-200 $\mathrm{mg} / \mathrm{kg} /$ day) to achieve beneficial effects of thalidomide, due to the short half-life of this drug [25]. In contrast, emphasis needs to be placed on the finding that thalidomide in the present study was effective in suppressing peritoneal fibrosis at a low dose $(10 \mathrm{mg} / \mathrm{kg} /$ day $)$ close to doses currently used in clinical situations. The anti-fibrotic effects of thalidomide seen with administration of a much lower dose could reasonably be considered likely to help in minimizing adverse effects and may lead to clinical applications in the future.

Since the capacity for ultrafiltration across the peritoneal membrane is well known as a major predictor of outcome and mortality in PD patients [3, 4], the efficacy of thalidomide on peritoneal function was examined using a transport study. CG treatment induced a major increase in permeability for creatinine, and this modification was in turn attenuated in the $\mathrm{CG}+$ thalidomide group. These data indicate that thalidomide treatment, through inhibition of VEGF, exerts not only anti-angiogenic effects, but also protective effects on the increase in peritoneal permeability in regard to peritoneal function.

In conclusion, these findings indicate that thalidomide might be useful in preventing the progression of peritoneal fibrosis and the alteration of peritoneal function by inhibiting both angiogenesis and collagen accumulation.

\section{Acknowledgments}

The authors wish to thank Dr. Satoshi Funakoshi for helpful advice. We also wish to thank Ms. Tomomi Kurashige and Ms. Ryoko Yamamoto for their excellent technical assistance. 


\section{References}

1. Abo, T., Nagayasu, T., Hishikawa, Y., Tagawa, T., Nanashima, A., Yamayoshi, T., Matsumoto, K., An, S. and Koji, T. (2010) Expression of keratinocyte growth factor and its receptor in rat tracheal cartilage: possible involvement in wound healing of the damaged cartilage. Acta Histochem. Cytochem. 43; 89-98.

2. Aroeira, L. S., Aguilera, A., Sanchez-Tomero, J. A., Bajo, M. A., del Peso, G., Jimenez-Heffernan, J. A., Selgas, R. and LopezCabrera, M. (2007) Epithelial to mesenchymal transition and peritoneal membrane failure in peritoneal dialysis patients: pathologic significance and potential therapeutic interventions. J. Am. Soc. Nephrol. 18; 2004-2013.

3. Brimble, K. S., Walker, M., Margetts, P. J., Kundhal, K. K. and Rabbat, C. G. (2006) Meta-analysis: peritoneal membrane transport, mortality, and technique failure in peritoneal dialysis. J. Am. Soc. Nephrol. 17; 2591-2598.

4. Brown, E. A., Davies, S. J., Rutherford, P., Meeus, F., Borras, M., Riegel, W., Divino Filho, J. C., Vonesh, E. and van Bree, M. (2003) Survival of functionally anuric patients on automated peritoneal dialysis: the European APD Outcome Study. J. Am. Soc. Nephrol. 14; 2948-2957.

5. Calabrese, L. and Fleischer, A. B. (2000) Thalidomide: current and potential clinical applications. Am. J. Med. 108; 487-495.

6. Coles, G. A. and Topley, N. (2000) Long-term peritoneal membrane changes. Adv. Ren. Replace. Ther. 7; 289-301.

7. Combet, S., Miyata, T., Moulin, P., Pouthier, D., Goffin, E. and Devuyst, O. (2000) Vascular proliferation and enhanced expression of endothelial nitric oxide synthase in human peritoneum exposed to long-term peritoneal dialysis. J. Am. Soc. Nephrol. 11; 717-728.

8. D'Amato, R. J., Loughnan, M. S., Flynn, E. and Folkman, J. (1994) Thalidomide is an inhibitor of angiogenesis. Proc. Natl. Acad. Sci. US A 91; 4082-4085.

9. Damavandi, E., Hishikawa, Y., Izumi, S., Shin, M. and Koji, T. (2002) Involvement of Bax redistribution in the induction of germ cell apoptosis in neonatal mouse testes. Acta Histochem. Cytochem. 35; 449-459.

10. Devuyst, O., Margetts, P. J. and Topley, N. (2010) The pathophysiology of the peritoneal membrane. J. Am. Soc. Nephrol. 21; 1077-1085.

11. Dvorak, H. F., Brown, L. F., Detmar, M. and Dvorak, A. M. (1995) Vascular permeability factor/vascular endothelial growth factor, microvascular hyperpermeability, and angiogenesis. Am. J. Pathol. 146; 1029-1039.

12. Ferrara, N. (2000) Vascular endothelial growth factor and the regulation of angiogenesis. Recent Prog. Horm. Res. 55; 15-35.

13. Gandhi, V. C., Humayun, H. M., Ing, T. S., Daugirdas, J. T., Jablokow, V. R., Iwatsuki, S., Geis, W. P. and Hano, J. E. (1980) Sclerotic thickening of the peritoneal membrane in maintenance peritoneal dialysis patients. Arch. Intern. Med. 140; 1201-1203.

14. Gavrieli, Y., Sherman, Y. and Ben-Sasson, S. A. (1992) Identification of programmed cell death in situ via specific labeling of nuclear DNA fragmentation. J. Cell Biol. 119; 493-501.

15. Honda, K., Nitta, K., Horita, S., Yumura, W. and Nihei, H. (1996) Morphological changes in the peritoneal vasculature of patients on CAPD with ultrafiltration failure. Nephron $72 ; 171-176$.

16. Io, H., Hamada, C., Ro, Y., Ito, Y., Hirahara, I. and Tomino, Y. (2004) Morphologic changes of peritoneum and expression of VEGF in encapsulated peritoneal sclerosis rat models. Kidney Int. 65; 1927-1936.

17. Ishii, Y., Sawada, T., Shimizu, A., Tojimbara, T., Nakajima, I., Fuchinoue, S. and Teraoka, S. (2001) An experimental sclerosing encapsulating peritonitis model in mice. Nephrol. Dial. Transplant. 16; 1262-1266.

18. Kim, K. K., Kugler, M. C., Wolters, P. J., Robillard, L., Galvez, M. G., Brumwell, A. N., Sheppard, D. and Chapman, H. A.
(2006) Alveolar epithelial cell mesenchymal transition develops in vivo during pulmonary fibrosis and is regulated by the extracellular matrix. Proc. Natl. Acad. Sci. US A 103; 13180-13185.

19. Lv, P., Luo, H. S., Zhou, X. P., Chireyath Paul, S., Xiao, Y. J., Si, X. M. and Liu, S. Q. (2006) Thalidomide prevents rat liver cirrhosis via inhibition of oxidative stress. Pathol. Res. Pract. 202; 777-788

20. Lv, P., Luo, H. S., Zhou, X. P., Xiao, Y. J., Paul, S. C., Si, X. M. and Zhou, Y. H. (2007) Reversal effect of thalidomide on established hepatic cirrhosis in rats via inhibition of nuclear factorkappaB/inhibitor of nuclear factor-kappaB pathway. Arch. Med. Res. 38; 15-27.

21. Lv, P., Paul, S. C., Xiao, Y., Liu, S. and Luo, H. (2006) Effects of thalidomide on the expression of adhesion molecules in rat liver cirrhosis. Mediators Inflamm. 2006; 93253.

22. Margetts, P. J., Oh, K. H. and Kolb, M. (2005) Transforming growth factor-beta: importance in long-term peritoneal membrane changes. Perit. Dial. Int. 25 Suppl 3; S15-17.

23. Mishima, Y., Miyazaki, M., Abe, K., Ozono, Y., Shioshita, K., Xia, Z., Harada, T., Taguchi, T., Koji, T. and Kohno, S. (2003) Enhanced expression of heat shock protein 47 in rat model of peritoneal fibrosis. Perit. Dial. Int. 23; 14-22.

24. Miyajima, K., Takekoshi, S., Itoh, J., Kakimoto, K., Miyakoshi, T. and Osamura, R. Y. (2010) Inhibitory effects of anti-VEGF antibody on the growth and angiogenesis of estrogen-induced pituitary prolactinoma in Fischer 344 rats: animal model of VEGF-targeted therapy for human endocrine tumors. Acta Histochem. Cytochem. 43; 33-44.

25. Mondello, S., Mazzon, E., Di Paola, R., Crisafulli, C., Mondello, P., Buemi, M., Aloisi, C. and Cuzzocrea, S. (2009) Thalidomide suppresses sclerosing encapsulating peritonitis in a rat experimental model. Shock 32; 332-339.

26. Ni, J., Verbavatz, J. M., Rippe, A., Boisde, I., Moulin, P., Rippe, B., Verkman, A. S. and Devuyst, O. (2006) Aquaporin-1 plays an essential role in water permeability and ultrafiltration during peritoneal dialysis. Kidney Int. 69; 1518-1525.

27. Nishino, T., Miyazaki, M., Abe, K., Furusu, A., Mishima, Y., Harada, T., Ozono, Y., Koji, T. and Kohno, S. (2003) Antisense oligonucleotides against collagen-binding stress protein HSP47 suppress peritoneal fibrosis in rats. Kidney Int. 64; 887-896.

28. Paravar, T. and Lee, D. J. (2008) Thalidomide: mechanisms of action. Int. Rev. Immunol. 27; 111-135.

29. Paul, S. C., Lv, P., Xiao, Y. J., An, P., Liu, S. Q. and Luo, H. S. (2006) Thalidomide in rat liver cirrhosis: blockade of tumor necrosis factor-alpha via inhibition of degradation of an inhibitor of nuclear factor-kappaB. Pathobiology 73; 82-92.

30. Scotton, C. J. and Chambers, R. C. (2007) Molecular targets in pulmonary fibrosis: the myofibroblast in focus. Chest $132 ; 1311-$ 1321.

31. Shirendeb, U., Hishikawa, Y., Moriyama, S., Win, N., Thu, M. M., Mar, K. S., Khatanbaatar, G., Masuzaki, H. and Koji, T. (2009) Human papillomavirus infection and its possible correlation with p63 expression in cervical cancer in Japan, Mongolia, and Myanmar. Acta Histochem. Cytochem. 42; 181-190.

32. Tabata, C., Tabata, R., Kadokawa, Y., Hisamori, S., Takahashi, M., Mishima, M., Nakano, T. and Kubo, H. (2007) Thalidomide prevents bleomycin-induced pulmonary fibrosis in mice. $J$. Immunol. 179; 708-714.

33. Williams, J. D., Craig, K. J., Topley, N., Von Ruhland, C., Fallon, M., Newman, G. R., Mackenzie, R. K. and Williams, G. T. (2002) Morphologic changes in the peritoneal membrane of patients with renal disease. J. Am. Soc. Nephrol. 13; 470-479.

34. Yndestad, A., Vinge, L. E., Bjornerheim, R., Ueland, T., Wang, J. E., Froland, S. S., Attramadal, H., Aukrust, P. and Oie, E. (2006) Thalidomide attenuates the development of fibrosis during postinfarction myocardial remodelling in rats. Eur. J. Heart. Fail. 8; 790-796. 
35. Yoshio, Y., Miyazaki, M., Abe, K., Nishino, T., Furusu, A., Mizuta, Y., Harada, T., Ozono, Y., Koji, T. and Kohno, S. (2004) TNP-470, an angiogenesis inhibitor, suppresses the progression of peritoneal fibrosis in mouse experimental model. Kidney Int. $66 ; 1677-1685$

36. Zhang, S., Li, M., Gu, Y., Liu, Z., Xu, S., Cui, Y. and Sun, B (2008) Thalidomide influences growth and vasculogenic mimicry channel formation in melanoma. J. Exp. Clin. Cancer Res. 27; 60.

37. Zweers, M. M., de Waart, D. R., Smit, W., Struijk, D. G. and Krediet, R. T. (1999) Growth factors VEGF and TGF-betal in peritoneal dialysis. J. Lab. Clin. Med. 134; 124-132.

38. Zweers, M. M., Struijk, D. G., Smit, W. and Krediet, R. T. (2001) Vascular endothelial growth factor in peritoneal dialysis: a longitudinal follow-up. J. Lab. Clin. Med. 137; 125-132.

This is an open access article distributed under the Creative Commons Attribution License, which permits unrestricted use, distribution, and reproduction in any medium, provided the original work is properly cited. 